\title{
On long-term modulation of the Sun's magnetic cycle
}

\author{
J. Beer, ${ }^{1}$ S. M. Tobias ${ }^{2 \star}$ and N. O. Weiss ${ }^{3}$ \\ ${ }^{1}$ EAWAG, Dübendorf, $\mathrm{CH}-8600$, Switzerland \\ ${ }^{2}$ Department of Applied Mathematics, University of Leeds, Leeds LS2 9JT, UK \\ ${ }^{3}$ Department of Applied Mathematics and Theoretical Physics, University of Cambridge, Cambridge CB3 OWA, UK
}

Accepted 2017 September 5. Received 2017 September 5; in original form 2017 May 26

\begin{abstract}
We utilize reconstructions based on cosmogenic radionuclides as well as direct observations of solar magnetic activity, to argue that the solar dynamo has operated similarly to the present day for at least the past $10000 \mathrm{yr}$. The persistence of the 87-yr Gleissberg cycle throughout supermodulation events suggests that the Hale and Schwabe cycles continue independently of the modulational mechanism for activity. We further analyse behaviour of solar activity during the Spörer and Maunder Minima. Such grand minima recur with the characteristic de Vries period of approximately $208 \mathrm{yr}$ but their incidence is modulated by the Hallstatt cycle with a characteristic period of around $2300 \mathrm{yr}$. We ascribe the latter to supermodulation, caused by breaking the symmetry of the dynamo pattern. Finally, we emphasize the need for further calculations in order to determine the effects of changes in solar field morphology and symmetry on the solar wind and on cosmic ray deflection.
\end{abstract}

Key words: dynamo-magnetic fields - sunspots.

\section{INTRODUCTION}

The 11-yr sunspot cycle (the Schwabe cycle) can be traced back to the early 17 th century from telescopic observations (Hathaway 2015; Rahmanifard et al. 2017; Usoskin 2017). Two hundred years later, it was demonstrated that it corresponds to an underlying magnetic cycle with a 22-yr period (the Hale cycle). Even the relatively short sunspot record shows that solar activity is far from being strictly periodic and that there are various patterns of longer time-scale modulation. Over the past two centuries, this record indicates the presence of regular modulation of the basic cycle with a period of around $90 \mathrm{yr}$. Specifically, Gleissberg $(1939,1955)$ pointed out that sunspot numbers (since 1750), as well as the mean displacement of sunspots from the equator (from 1857 to 1954) have a modulational period of approximately $90 \mathrm{yr}$. Further obvious evidence of modulation of the basic cycle arises from the presence of the Maunder Minimum in the sunspot record, for solar activity was dramatically reduced between 1645 and 1710, and also of the Dalton Minimum of the early 19th century with a (less significant) drop in activity (Beer, McCracken \& von Steiger 2012; Usoskin 2017).

Evidence for modulation on much longer time-scales is derived from proxy data. Variations of solar activity lead to modulation of the flux of galactic cosmic rays entering the terrestrial atmosphere (Beer et al. 2012; Potgieter 2013) and so of the neutrons generated as they impinge on the earth's upper atmosphere, as shown in Fig. 1. Hence, there is a corresponding variation in the consequent production of radionuclides such as ${ }^{10} \mathrm{Be}$ and ${ }^{14} \mathrm{C}$. Detailed analysis of the abundances of these radionuclides, in ice cores and tree rings, respectively, has revealed a number of significant results (Beer et al. 2012; McCracken et al. 2013a; Usoskin 2017). The first of these is that events such as the Maunder Minimum (known as grand minima) recur with a well-defined mean period (the de Vries period) of approximately $208 \mathrm{yr}$, as first pointed out on the basis of analyses of ${ }^{14} \mathrm{C}$ abundances in bristle-cone pines (de Vries 1958; Suess 1965). Interestingly, although the number of sunspots is reduced during these episodes, the radionuclide data demonstrate the presence of well-defined cycles throughout the Maunder Minimum and through most of the preceding Spörer Minimum. Secondly, further analysis of the radionuclide record indicates the presence of supermodulation of the basic solar cycle (Weiss \& Tobias 2016). The long-term record of solar activity shows that two distinct patterns of behaviour are possible. In the first pattern, recurrent deep grand minima occur regularly and modulation of the basic cycle is severe; in the second pattern, there are no deep minima (McCracken et al. 2013b). A key observation for this paper is that frequency analysis of the radionuclide record demonstrates that in both patterns of behaviour a clear signal with a period of approximately $87 \mathrm{yr}$ is apparent over the last $10000 \mathrm{yr}$ (McCracken et al. 2013a,b).

The spatio-temporal character of the Sun's magnetic field is often best brought out by the famous butterfly diagram, showing the latitudinal dependence of sunspots as a function of time (e.g. Hathaway 2015). The spots appear as a wave of magnetic activity progressing from mid-latitudes both polewards and towards the equator, with the cycle dying out as the wave reaches the equator. 


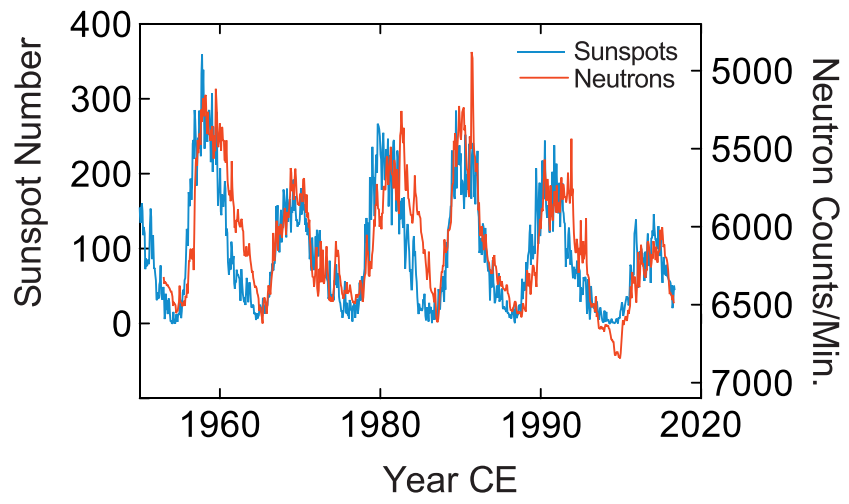

Figure 1. Comparison between monthly sunspot numbers (from SILSO ISN, v. 2.0, and shown in blue) and the monthly neutron count rates (shown in red; note the inverted scale) from the Climax (1953-2006) and Oulu (2006-2013, see http://cosmic rays.oulu.fi) Observatories.

For stronger cycles, the waves start at somewhat higher latitudes. Generally the wings of the butterfly are largely symmetric about the equator (though the predominantly toroidal fields linking sunspot pairs have opposite polarities in each hemisphere - leading to a largely antisymmetric large-scale toroidal field). This azimuthally averaged magnetic field is therefore predominantly dipolar. There was however a brief episode (ca 1760) when sunspots first appeared at the equator (Arlt 2009), indicating that the Sun's field was quadrupolar. Even earlier there was an interval (as the Sun emerged from the Maunder Minimum) when sunspots were almost exclusively found in the Southern hemisphere (Ribes \& NesmeRibes 1993; Arlt \& Weiss 2014) and so the dipole and quadrupole fields were both present in a so-called mixed mode, corresponding to a hemispheric magnetic field. The two solar hemispheres are apparently strongly (though imperfectly) coupled. ${ }^{1}$

In the next section, we focus on recent measurements of ${ }^{10} \mathrm{Be}$ abundances in ice cores from Greenland and the variations in solar magnetic activity which can be deduced from them (McCracken \& Beer 2015; Usoskin 2017). These results, spanning an interval of over five centuries, can be contrasted with telescopic observations of sunspots since the time of Galileo, and then extended back so that they cover a total of two grand minima. Here, we look at details of the relationship between the sunspot and radionuclide records in order to investigate how cycles are altered during grand minima.

In Section 3, we turn to the ${ }^{14} \mathrm{C}$ record which spans the past 10000 yr (Reimer et al. 2013; Muscheler, Adolphi \& Knudsen 2014; Usoskin 2017) and focus our attention first on the well-attested de Vries cycle. Previous studies have applied Fourier analysis to both the ${ }^{10} \mathrm{Be}$ and ${ }^{14} \mathrm{C}$ records in order to pick out various periodicities (Beer et al. 2012; McCracken et al. 2013b). We shall take an alternative approach, following packets of frequencies corresponding to the periodicities identified by McCracken et al. (2013b). This provides a clearer (though necessarily filtered) picture of the nonlinear oscillations of the Sun's magnetic field, which complements the earlier results. We demonstrate this approach by applying it not only to the 208-yr de Vries cycle but also to the circa 2300-yr modulation (the so-called Hallstatt cycle) of that cycle.

\footnotetext{
${ }^{1}$ Usoskin et al. (2009) describe the appearance of a sparse pattern of sunspots during the interval 1793-1796, with activity concentrated in the Northern (rather than the Southern) hemisphere. This anomalous hemispheric (mixed-mode) pattern replaces the so-called missing cycle before the Dalton minimum.
}

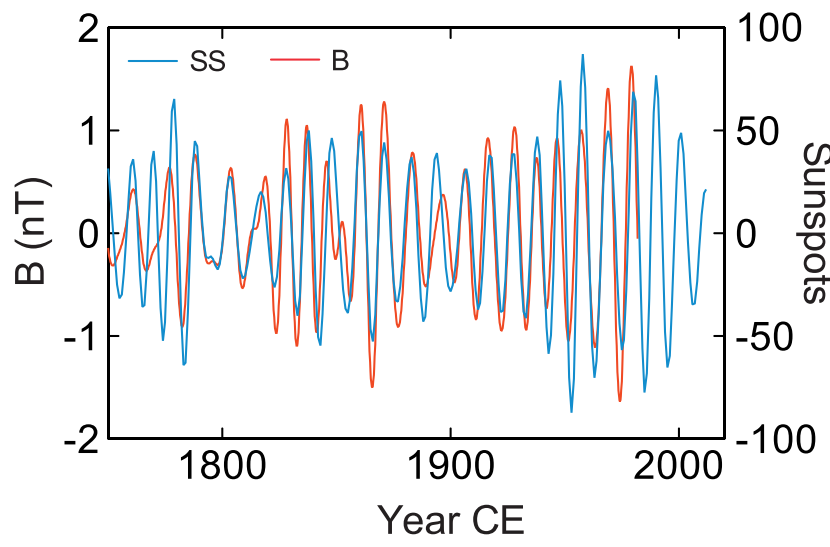

Figure 2. Comparison between monthly sunspot numbers (in blue) and values of the solar interplanetary magnetic field derived from a combination of the NGRIP and Dye $3{ }^{10} \mathrm{Be}$ records from Greenland (shown in red) for the interval from $1750 \mathrm{CE}$ to the present (McCracken \& Beer 2015). Both data sets were processed the same way by applying a band-pass filter (7.46-15.75 yr).

Next, in Section 4, we study the variable but persistent Gleissberg cycle with a mean period of $87 \mathrm{yr}$. We can exploit it to argue that the underlying solar dynamo, with its Hale magnetic cycle and its Schwabe activity cycle, has continued to operate much as now, at least throughout the last $10000 \mathrm{yr}$ which make up most of the postglacial Holocene epoch on Earth. Then, in Section 5, we discuss the role of symmetries in the solar dynamo, and argue that the persistence of the 87-yr Gleissberg period confirms that the Schwabe and Hale periodicities must have been present for at least the last two millennia and probably for a total of $50000 \mathrm{yr}$. Our results are then summarized in the concluding section, where we emphasize that a deeper understanding of the effects of symmetry and morphology of the field on the solar wind is needed.

\section{THE 10BE RECORD}

The sunspot record, extending back for $400 \mathrm{yr}$, can be compared with direct measurements of the modulation over the last few decades of the neutron flux generated by cosmic rays impinging on the earth's upper atmosphere. Fig. 1 contrasts the monthly sunspot numbers with monthly neutron count rates, which are available for the last six solar cycles. It is abundantly clear that (over this brief interval) the neutron count rate is in close agreement with the sunspot record. Since radionuclides such as ${ }^{10} \mathrm{Be}$ and ${ }^{14} \mathrm{C}$ are produced by nuclear reactions involving secondary cosmic ray protons and neutrons, it is only to be expected that the ${ }^{10} \mathrm{Be}$ abundances in polar ice cores should likewise provide a record of solar activity which concurs with the sunspot records. Fortunately, a record with annual resolution, extending back for $600 \mathrm{yr}$ to $1389 \mathrm{CE}$, has recently been provided by the North Greenland Ice Project (Berggren et al. 2009). This record could be compared with data from a second ice core at Dye 3 in southern Greenland (Beer, Tobias \& Weiss 1998) after the latter record had been corrected and recalibrated (McCracken $\&$ Beer 2015). The composite annual record of ${ }^{10} \mathrm{Be}$ production, obtained by combining data from these two sets of ice cores, can then be inverted and transformed to represent the normalized magnetic field. Fig. 2 shows a comparison between this record of solar activity and the sunspot record from 1750 to the present. Both records are appropriately filtered to eliminate short- and long-term fluctuations and to focus on the 11-yr Schwabe cycle. Again it is clear that 


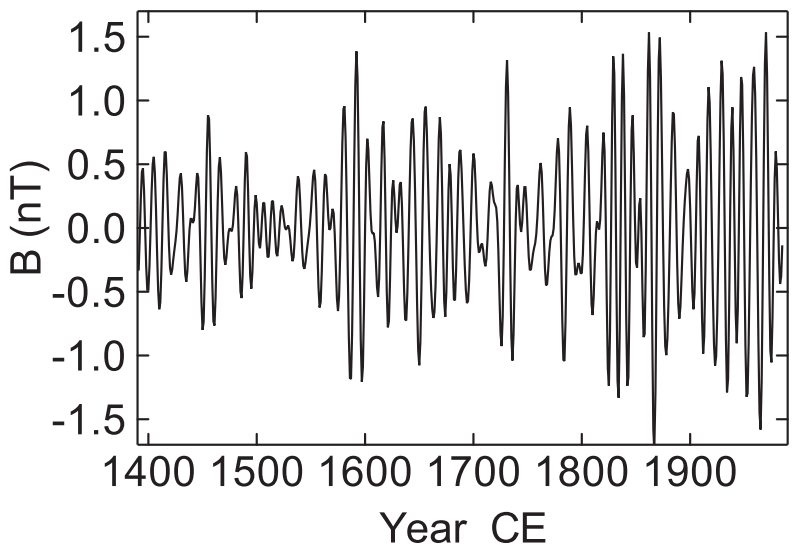

Figure 3. As Fig. 2 but showing only the interplanetary magnetic field derived from the two ${ }^{10} \mathrm{Be}$ records, now for the full available range from $1391 \mathrm{CE}$ to the present. A band filter (7.46-15.75 yr) has again been applied.

cyclic behaviour prevails in both records; indeed, all maxima (with the exception of that at $1770 \mathrm{CE}$ ) are suitably aligned. The maxima of the two data sets are in fair quantitative agreement.

Discrepancies become more apparent in the fuller record shown in Fig. 3. Sunspots have been observed through telescopes since 1610 but their incidence was low during the Maunder Minimum, from 1645 to $1710 .^{2}$ Scarcely any spots were seen between 1660 and 1670, while precise measurements at the Paris Observatory between 1670 and 1710 revealed an abnormal pattern (Ribes \& Nesme-Ribes 1993; Arlt \& Weiss 2014; Usoskin 2017) again with relatively few spots, almost all confined to the Southern hemisphere of the Sun. Thus, the sunspot record for this interval indicates only extremely weak (and asymmetrically distributed) activity. Yet the ${ }^{10} \mathrm{Be}$ record in Fig. 3 shows moderate (though declining) activity through the latter part of the 17th century, with the weakest cycle around 1710 , after the spots had actually returned. So there seems to be a discrepancy between the telescopic record and the radionuclide measurements. This, we believe, is important and is extremely suggestive that the production rates of ${ }^{10} \mathrm{Be}$ (and presumably other cosmogenic radionuclides) may be sensitive not only to the amplitude of solar activity, as measured by sunspot number, but also to the symmetry, and perhaps the morphology, of the underlying magnetic field causing the modulation. ${ }^{3}$ We return to this point in the discussion. On the other hand, during the Spörer Minimum in the 16th century (when no direct observations of sunspots are available) there was an interval around 1520 with extremely low activity. We may infer from the ${ }^{10} \mathrm{Be}$ isotopic record, therefore, that the Spörer Minimum was much deeper than the Maunder Minimum which followed it, as well as having a longer duration. That is indeed consistent with conclusions reached by Stuiver \& Quay (1980, see

\footnotetext{
${ }^{2}$ Detailed studies (Owens, Usoskin \& Lockwood 2012) suggest that the rate of loss of open solar magnetic flux follows variations in the tilt of the heliospheric current sheet in such a way that fluctuations in the heliospheric modulation potential vary out of phase with the sunspot cycle during a grand minimum.

${ }^{3}$ Here, it should be noted that during periods of strong solar activity the low-energy part of the differential energy spectrum of cosmic ray particles is considerably reduced. This leads to changes with smaller amplitudes than those which occur during a grand minimum period, when the same modulation has a larger effect on the amplitude. This arises owing to the non-linearity of the relation between solar activity and the production of cosmogenic radionuclides (Poluianov, Usoskin \& Kovaltsov 2014).
}

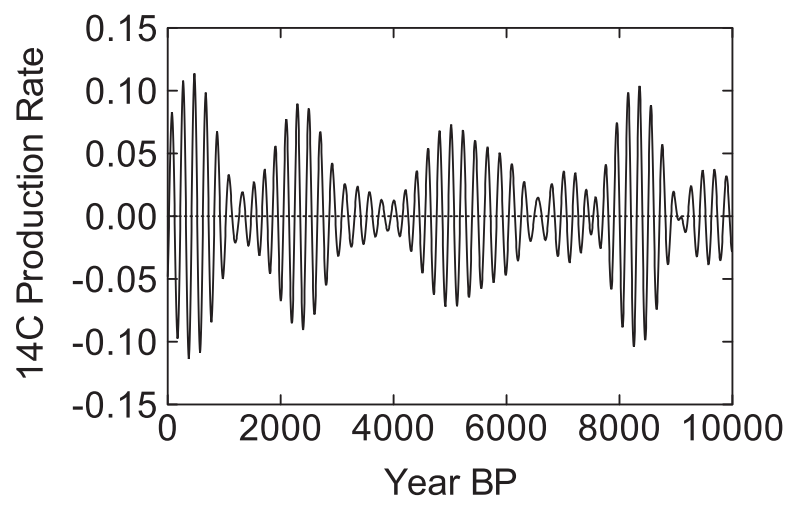

Figure 4. The $\mathrm{P}^{14} \mathrm{C}$ record of the radiocarbon production rate, after bandpass filtering with cut-offs at $1 / 230$ and $1 / 180 \mathrm{yr}^{-1}$, so as to emphasize the 208-yr de Vries cycle. This record shows an ordered structure with several episodes of regular activity separated by intervals during which activity is reduced; the maxima in this radionuclide record actually correspond to grand minima in solar activity. This modulation is further modulated with a characteristic time-scale of around $2300 \mathrm{yr}$ which is associated with the so-called Hallstatt cycle.

also Stuiver \& Braziunas 1988; Usoskin 2017) on the basis of ${ }^{14} \mathrm{C}$ abundances in tree rings.

\section{THE ${ }^{14}$ C RECORD AND THE 208-YR DE VRIES CYCLE}

For the next two sections, we rely on the IntCal13 record of radiocarbon abundances $\Delta^{14} \mathrm{C}$ measured in tree rings between 0 and $10000 \mathrm{yr}$ BP, where BP denotes dates prior to $1950 \mathrm{CE}$ (Reimer et al. 2013). The advantage of using the ${ }^{14} \mathrm{C}$ data is that the timescale is based on tree ring counting and therefore very reliable. The temporal resolution of the data is $5 \mathrm{yr}$. To correct for the damping effect of the carbon cycle the $\Delta^{14} \mathrm{C}$ data have been converted into production data $\mathrm{P}^{14} \mathrm{C}$ (Muscheler et al. 2014); this conversion only affects the amplitudes of the spectral lines. Long-period end effects have been partially suppressed by neglecting results for ages greater than $10000 \mathrm{yr}$ in analysis of the original data; the large amplitude variations at the beginning of the record may, however, be exaggerated owing to the relevant end effect.

\subsection{The 208-yr de Vries cycle}

Our discussion focuses first on the well-established de Vries (or Suess) cycle, with a precisely determined mean period of $208 \mathrm{yr}$ (McCracken et al. 2013a,b). Fig. 4 shows the radiocarbon record, filtered so as to emphasize this periodicity. As expected, the de Vries cycle is very strikingly displayed: there is a characteristic modulation with an average period of $208 \mathrm{yr}$, giving rise to alternate grand maxima and grand minima in activity. (Note that the ${ }^{14} \mathrm{C}$ abundances are anticorrelated with the Sun's magnetic activity, so that maxima in this image actually correspond to grand minima in solar activity.) This pattern itself is modulated on a longer timescale, as discussed below. ${ }^{4}$

In Fig. 5, we contrast two versions of the $\mathrm{P}^{14} \mathrm{C}$ record: the first presents this record after it has been high-pass filtered with a cut-off

\footnotetext{
${ }^{4}$ An alternative representation of the data, showing amplitude versus period and time, is presented by McCracken et al. (2013b); see also Abreu et al. (2012) and Usoskin et al. (2016).
} 


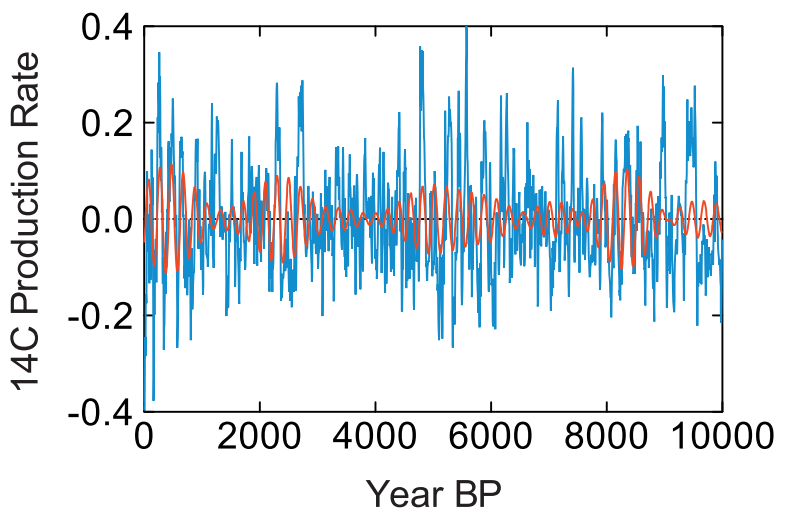

Figure 5. A high-pass record of the radiocarbon production rate $\mathrm{P}^{14} \mathrm{C}$ (in blue), showing only periods which are less than 1000 yr. Once again, the apparent maxima actually correspond to deep grand minima, with shorter lifetimes than shown in Fig. 4. This record is contrasted with that from the previous figure (in red), where the sharp extrema have been smoothed out. The modulation again exhibits the characteristic time-scale associated with the Hallstatt cycle, but the actual record is far more jagged than the smoothed version: the grand extrema appear much sharper and more extreme (especially the very deep grand minima).

at a period of $1000 \mathrm{yr}$, while the second repeats the band-pass filtered record from Fig. 4. These two records are clearly correlated, for the largest fluctuations in the high-pass record coincide with extrema in the de Vries cycle. However, the procedure adopted in constructing Fig. 4 smoothed out the narrow peaks and troughs which are shown in Fig. 5, especially the slender upward spikes that correspond to grand minima: yet we know that these extrema indicate the presence of global bifurcations that are in fact responsible for the complicated dynamical behaviour of the whole system. Detailed studies (see, for example, Tobias \& Weiss 2007a,b; Weiss \& Tobias 2016) confirm that such bifurcations not only facilitate the appearance of chaotic behaviour but also permit transitions between dipole and quadrupole symmetries, as well as allowing the appearance of mixed-mode patterns that lack either symmetry.

\subsection{The 2300-yr Hallstatt modulation}

A striking feature of the long-term record from radionuclides is the appearance not only of modulation by grand minima (of which the Maunder Minimum is the first example) but also of intervals in which grand minima are absent. The transition from one form of modulation to the other, which we have termed supermodulation, is associated with the Hallstatt period of around $2300 \mathrm{yr}$ (Vasiliev \& Durgachev 2002; Usoskin et al. 2016; Weiss \& Tobias 2016).

Interestingly, the overall pattern in Fig. 5 scarcely changes as the cut-off period is progressively increased, from 1000 to $4000 \mathrm{yr}$, as illustrated by the red and blue curves in Figs 5 and 6, implying that the Hallstatt period provides an upper bound for significant periodicities associated with the solar dynamo. In Fig. 7, with an effective cut-off period of $10000 \mathrm{yr}$, we see a very different pattern of long-period modulation which is no longer related to the Hallstatt cycle: instead, the record varies with a period of around $8000 \mathrm{yr}$. This effect we interpret as a consequence of terrestrial processes that are not directly related to the solar dynamo, but are predominantly associated with variations of the geomagnetic field (Nilsson et al. 2014), augmented perhaps by changes in the carbon cycle.

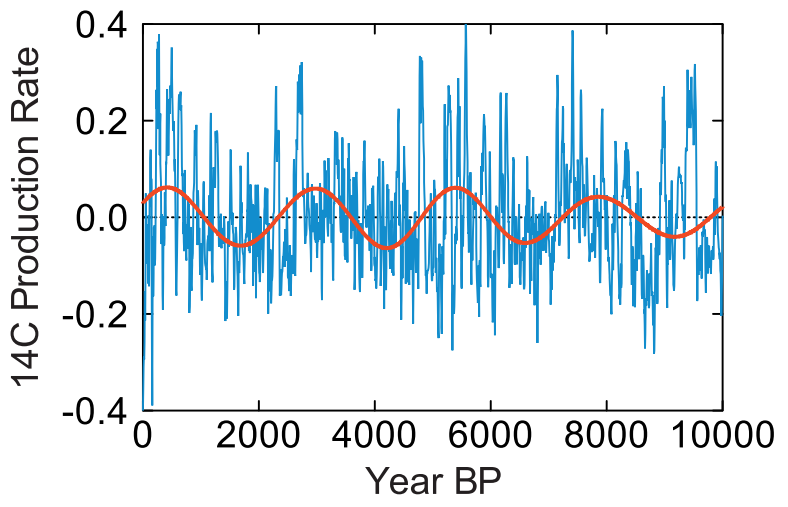

Figure 6. The $\mathrm{P}^{14} \mathrm{C}$ record after high-pass filtering with a cut-off at a period of $4000 \mathrm{yr}$ (the blue curve), contrasted with the band-pass-filtered record for periods between 1800 and $2600 \mathrm{yr}$ (the red curve) so as to emphasize the Hallstatt cycle. This record shows an ordered structure with several episodes of regular activity separated by intervals during which activity is reduced; once again, the maxima here actually correspond to minima in solar activity. Such modulation exhibits the characteristic time-scale of around $2300 \mathrm{yr}$ for the supermodulation which is associated with the Hallstatt cycle.

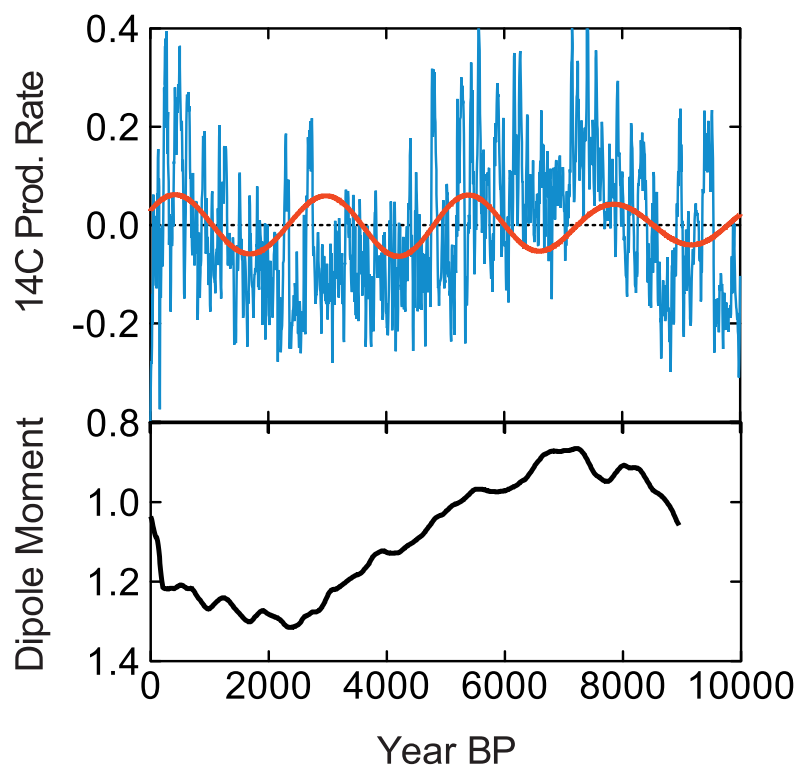

Figure 7. Upper panel: the Hallstatt cycle, represented again by the $\mathrm{P}^{14} \mathrm{C}$ record after band-pass filtering with cut-offs at periods of 2600 and $1800 \mathrm{yr}$ (red curve) contrasted now with the full unfiltered $\mathrm{P}^{14} \mathrm{C}$ record (blue curve). There is clearly no close correspondence between the two data sets. The full record shows modulation with a characteristic half-period of around $4000 \mathrm{yr}$ which is predominantly associated with geomagnetic variability. Lower panel: the variation with time of the Earth's magnetic dipole moment during most of the Holocene period (after Nilsson et al. 2014). Comparison of the two panels confirms that the long-term variation of the radionuclide record is driven by changes in the Earth's magnetic field.

\section{SYMMETRY-BREAKING AND THE 87-YR GLEISSBERG CYCLE}

We now turn to a weaker effect. Fig. 8 shows the radiocarbon record after it has been filtered so as to demonstrate the persistency of the irregular Gleissberg cycle, with a well-defined mean period of 


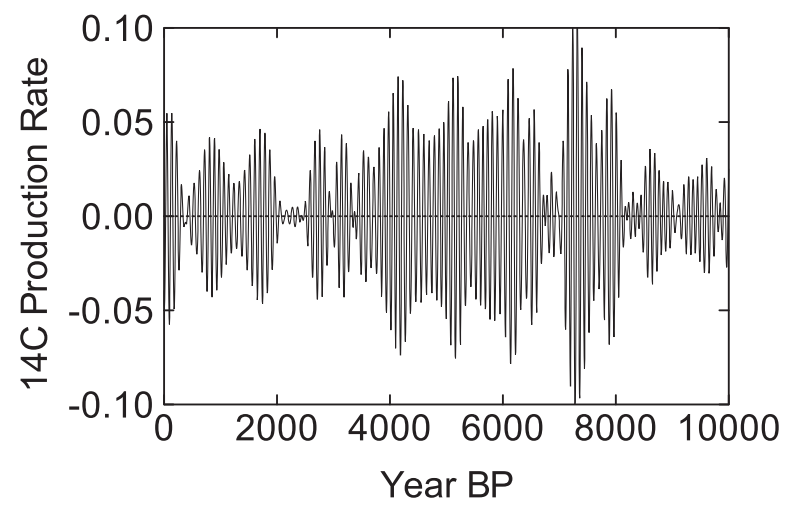

Figure 8. $10000 \mathrm{yr}$ of ${ }^{14} \mathrm{C}$ production rate $\left(\mathrm{P}^{14} \mathrm{C}\right)$, derived from $\Delta^{14} \mathrm{C}$ measurements on tree rings (IntCal13; Reimer et al. 2013) using a carbon cycle model (Muscheler et al. 2014). A band-pass filter with cut-offs at 1/100 and $1 / 75 \mathrm{yr}^{-1}$ has been applied to the ${ }^{14} \mathrm{C}$ production rate to emphasize the 87-yr Gleissberg cycle. This cycle has persisted for $10000 \mathrm{yr}$, while its amplitude varies aperiodically, dropping occasionally to very low values. Some end effects have been removed. The fluctuations vary considerably in amplitude but it is difficult to discern any systematic pattern.

$87 \mathrm{yr}^{5}{ }^{5}$ This cycle varies significantly in amplitude and occasionally almost completely disappears. There are prolonged episodes with large-amplitude fluctuations, and these are of variable duration. This behaviour appears to be chaotically modulated and unrelated to any other cyclic pattern, Nevertheless, these results do demonstrate that the Gleissberg cycle has indeed been a persistent feature of solar activity for at least the past 10000 years, though the Gleissberg record does not correlate with the $\mathrm{P}^{14} \mathrm{C}$ production rate record (see Fig. 9; Peristykh \& Damon 2003). What can be the cause of this effect?

Even when the solar field is at first sight largely dipolar, quadrupolar fields may play an important role, and mixed-mode fields break symmetries about the equator, as discussed by Weiss \& Tobias (2016); see also DeRosa, Brun \& Hoeksema (2012). Of particular interest for determining the origin of the $87-\mathrm{yr}$ periodicity is the observation that the Sun's 'magnetic equator' has recently wobbled with a period of approximately $90 \mathrm{yr}$ (e.g. Hathaway 2015). Pulkkinen et al. (1999) and Brooke et al. (2002) calculated the average latitudes of sunspots in the Northern and Southern hemispheres of the Sun between 1853 and 1996 and demonstrated that the difference between each hemisphere in that average latitude oscillates with this period. This is consistent with calculations that provide timeseries of the simpler statistic of the predominance $(\mathrm{N}-\mathrm{S})$ of numbers of spots in the Northern hemisphere $(\mathrm{N})$ over those in the Southern hemisphere (S), and they also show this period. Moreover, it is apparent that this period is very close not only to that identified by Gleissberg and others (e.g. Deng et al. 2016) but also to that which appears robustly in the longer term ${ }^{10} \mathrm{Be}$ and ${ }^{14} \mathrm{C}$ data in both patterns of the supermodulation as described above.

The correspondence of these two periods is surprising, as one might expect the Gleissberg period to be half that of the wobble of the magnetic equator, if the changes in radionuclide production were

\footnotetext{
${ }^{5}$ There are some indications that this periodicity is actually a combination of two closely related cycles, with periods of 86 and $88 \mathrm{yr}$ (McCracken et al. 2013a,b). Although other studies only recognize a broad-band with a typical time-scale (Gleissberg 1971; Ogurtsov et al. 2002), the detailed treatment - spanning $9400 \mathrm{yr}$ - by McCracken et al. (2013b) reveals an $87-y r$ periodicity that is quite sharply defined.
}

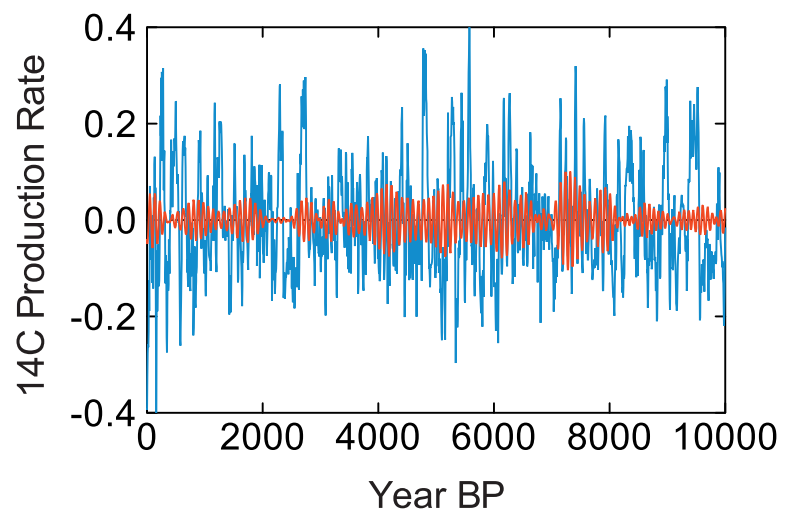

Figure 9. The Gleissberg record shown in Fig. 8 (red curve) is contrasted here with the $\mathrm{P}^{14} \mathrm{C}$ production rate record, after the latter has undergone filtering with a cut-off at a period of $1000 \mathrm{yr}$ (blue curve). There is no apparent correlation between these two records.

all due to changes in amplitude of the solar cycle while it retained equatorial antisymmetry. It is the origin of both this wobble and the discrepancy in periods which we address below.

\section{SYMMETRIES AND DYNAMOS}

The solar magnetic field is believed to be generated by a non-linear hydromagnetic dynamo, and some recent numerical models, relying on massive computation, have succeeded in reproducing various essential features of this process (e.g. Augustson et al. 2015; Passos, Charbonneau \& Miesch 2015; Raynaud \& Tobias 2016). While it is still fair to say that no precise universally accepted description of solar dynamo action yet exists, it is clear that symmetries play a key role in determining the dynamics of the field. It is often convenient to decompose the azimuthally averaged solar magnetic field into toroidal and poloidal components. In this axisymmetric geometry, the toroidal fields are azimuthal, whilst the poloidal fields are meridional. The linear problem admits axisymmetric fields of two symmetry classes; dipoles, where the toroidal field is antisymmetric about the equator (while the poloidal field is symmetric), and quadrupoles, which have symmetric toroidal fields and antisymmetric poloidal fields. Symmetry breaking occurs through the non-linear interactions of these two classes of fields, leading to the generation of mixed modes. Such non-linear interactions may be complicated and can only be understood via the application of bifurcation theory. There is a wide literature describing the possibilities (e.g. Jennings \& Weiss 1991; Knobloch, Tobias \& Weiss 1998; Tobias 2002; Weiss \& Tobias 2016). In the last of these papers, we have claimed that supermodulation arises through the transition to a mixed-mode pattern that lacks either perfect dipole or perfect quadrupole symmetry; such behaviour is seen in solutions of the full partial differential equations (Raynaud \& Tobias 2016).

We argue here that, because the $87-\mathrm{yr}$ period is revealed so strongly in both phases of the supermodulation cycle, this period cannot be associated with the particular type of modulational process - rather it must be closely linked with the basic dynamo cycle. It is immediately apparent that this 87-yr periodicity corresponds to eight Schwabe cycles and four Hale cycles, confirming that the Hale and Schwabe cycles must have persisted through all phases of modulation and supermodulation (Peristykh \& Damon 2003). We stress again that analysis of the sunspot records indicates that the $87-y r$ period is associated with a built-in symmetry breaking. To be sure, this has only been determined explicitly 
for a limited data set, and it is clearly of interest to extend the calculation of the location of the magnetic equator to longer and more complete records. Nevertheless, the presence of the $87-\mathrm{yr}$ periodicity in both the ${ }^{10} \mathrm{Be}$ and the ${ }^{14} \mathrm{C}$ radionuclide data demonstrates that it is a persistent feature of solar magnetic activity. Thus, a significant deduction from the radionuclide records is that both the underlying 11-yr Schwabe activity cycle and the 22-yr Hale magnetic cycle must have continued to operate at least throughout the $10^{4}$-yr period during which reliable evidence is currently available. The modulation of these cycles has, however, varied.

It is clear that the $10000-y r$ record of solar activity which is described both here and by McCracken et al. (2013b) actually displays two forms of modulation of the basic cycle. In the first, the regular pattern of activity is interrupted by repeated grand minima that recur, on average, with a well-defined period of $208 \mathrm{yr}$ (the de Vries period). In the second, which we have associated with supermodulation, grand minima are lacking although (as explained above) the 87 -yr modulation still survives. The aperiodic transitions from one form to the other are associated with the Hallstatt period (Usoskin et al. 2016).

The de Vries cycle reappears in several intervals of the GRIP ice core from Greenland: from approximately $22500 \mathrm{BP}$ to 10000 BP (Adolphi et al. 2014) and again from $50000 \mathrm{BP}$ to $25000 \mathrm{BP}$ (Wagner et al. 2001). Since the 208-yr periodicity is accompanied by an 87-yr modulation in the records studied by McCracken et al. (2013b), and the 87-yr modulation must (as we have argued above) itself be associated with the Schwabe and Hale cycles, we may interpret this evidence as suggesting that the 11-yr and 22-yr periodicities have persisted for at least 50 millennia.

\section{DISCUSSION}

These observational results prompt us to speculate on changes in the dynamo pattern that may be associated with grand minima. Apparently the cyclic dynamo carries on much as usual, generating weaker open field patterns that spread out and scatter cosmic ray particles throughout the heliosphere and not just near the earth's orbit. The field at the photosphere has to be predominantly poloidal, as it is ineffectual at producing sunspots. Yet the oscillatory dynamo functions all the same, and requires interactions between poloidal and toroidal fields within the Sun. So we infer that relatively strong toroidal fields are still present within the convection zone, but that they are pumped inwards and so rendered less effectual at the surface. These fields therefore have to be sufficiently weak to be carried downwards by the effects of turbulent convection combined with the layered meridional circulation which has been revealed both by helioseismology and by computational modelling (cf. Weiss \& Tobias 2016). In this way, the convection zone can act as a filter for the toroidal field and so change the ratio of poloidal to toroidal fields at the solar surface and hence the field morphology there, as suggested by Tobias et al. (1998, 2001). Nevertheless, the surviving fields near the surface have to be strong enough to distort the solar wind and so to affect the continuing deflection of cosmic ray protons. Further studies are clearly needed to strengthen these conjectures.

The results presented above immediately introduce a number of significant issues. The presence of the $87-y r$ period and its association with changes in the symmetry of the field raises the following question: what is the effect on the solar wind, and therefore on the production of cosmogenic radionuclides, of changes in the symmetry of the large-scale solar field, even if there is no change in its amplitude? A change in symmetry from a dipole field to a quadrupole of the same energy would tend to decrease the equatorial poloidal field and hence the field in the plane of the ecliptic. This would clearly deflect the solar wind and lead to a signal in the modulation of cosmic rays, and therefore in the production of cosmogenic radionuclides. A transition to a mixed-mode field or, in an extreme case, to a hemispheric field (Weiss \& Tobias 2016) would have comparable consequences. Detailed calculations of the amplitudes and even the signs of these effects are therefore desirable. We conclude by asking the important and interesting question 'How much of the variability of the production rates of cosmogenic radionuclides should be ascribed to variations in the amplitude of the solar cycle and how much to changes in its symmetry?'.

\section{ACKNOWLEDGEMENTS}

We have benefitted from constructive discussions with Rainer Arlt, and we thank the referee for suggesting many significant improvements to our paper.

\section{REFERENCES}

Abreu J. A., Beer J., Ferriz-Mas A., McCracken K. G., Steinhilber F., 2012, A\&A, 548, A88

Adolphi F. et al., 2014, Nat. Geosci., 7, 662

Arlt R., 2009, Sol. Phys., 255, 143

Arlt R., Weiss N. O., 2014, Space Sci. Rev., 186, 525

Augustson K., Brun A. S., Miesch M., Toomre J., 2015, ApJ, 809, 149

Beer J., Tobias S. M., Weiss N. O., 1998, Sol. Phys., 181, 237

Beer J., McCracken K. G., von Steiger R., 2012, Cosmogenic Radionuclides: Theory and Applications in the Terrestrial and Space Environments. Springer, Berlin

Berggren A.-M. et al., 2009, Geophys. Res. Lett., 36, L11801

Brooke J. M., Pelt J., Pulkkinen P., Tuominen I., 2002, in Rickman H., ed., Highlights of Astronomy, Vol. 12, as presented at the XXIVth General Assembly of the IAU - 2000. San Francisco, CA, Astronomical Society of the Pacific, p. 334

de Vries H., 1958, Proc. K. Ned. Akad. Wet. B, 61, 94

Deng L. H., Xiang Y. Y., Qu Z. N., An J. M., 2016, AJ, 151, 70

DeRosa M. L., Brun A. S., Hoeksema J. J., 2012, ApJ, 757, 96

Gleissberg W., 1939, Observatory, 62, 158

Gleissberg W., 1955, Z. Astrophys., 37, 108

Gleissberg W., 1971, Sol. Phys., 21, 40

Hathaway D. H., 2015, Living Rev. Sol. Phys., 12, 4

Jennings R. J., Weiss N. O., 1991, MNRAS, 252, 249

Knobloch E., Tobias S. M., Weiss N. O., 1998, MNRAS, 297, 1123

McCracken K. G., Beer J., 2015, Sol. Phys., 290, 3051

McCracken K. G., Beer J., Steinhilber F., Abreu J., 2013a, Space Sci. Rev., 176,59

McCracken K. G., Beer J., Steinhilber F., Abreu J., 2013b, Sol. Phys., 286, 609

Muscheler R., Adolphi F., Knudsen M. F., 2014, Quat. Sci. Rev., 106, 81

Nilsson A., Holme R., Korte M., Suttie N., Hill M., 2014, Geophys. J. Int., 198, 229

Ogurtsov M. G., Nagovitsyn Yu. A., Ocharov G. E., Jungner H., 2002, Sol. Phys., 211, 371

Owens M. J., Usoskin I., Lockwood M., 2012, Geophys. Res. Lett., 39, L19102

Passos D., Charbonneau P., Miesch M., 2015, ApJ, 800, L18

Peristykh A. N., Damon P. E. J., 2003, Geophys. Res., 108, 1003

Poluianov S. V., Usoskin I. G., Kovaltsov G. A., 2014, Sol. Phys., 289, 4701

Potgieter M. S., 2013, Living Rev. Sol. Phys., 10, 3

Pulkkinen P. J., Brooke J., Pelt J., Tuominen I., 1999, A\&A, 341, L43

Rahmanifard F. et al., 2017, ApJ, 837, 165

Raynaud R., Tobias S. M., 2016, J. Fluid Mech., 799, R6-1

Reimer P. J. et al., 2013, Radiocarbon, 55, 1869

Ribes J. C., Nesme-Ribes E., 1993, A\&A, 276, 549 
Stuiver M., Braziunas T. F., 1988, Nature, 338, 405

Stuiver M., Quay P. D., 1980, Science, 207, 11

Suess H. E., 1965, J. Geophys. Res., 70, 5937

Tobias S. M., 2002, Astron. Nachr., 323, 417

Tobias S. M., Weiss N. O., 2007a, in Dormy E., Soward A. M., eds, Mathematical Aspects of Natural Dynamos. Grenoble Sciences/CRC Press, Boca Raton, p. 281

Tobias S. M., Weiss N. O., 2007b, in Hughes D., Rosner R., Weiss N., eds, The Solar Tachocline. Cambridge Univ. Press, Cambridge, p. 319

Tobias S. M., Brummell N. H., Clune T. L., Toomre J., 1998, ApJ, 502, L177

Tobias S. M., Brummell N. H., Clune T. L., Toomre J., 2001, ApJ, 549, 1183
Usoskin I. G., 2017, Living Rev. Sol. Phys., 14, 3

Usoskin I. G. et al., 2009, ApJL, 700, L154

Usoskin I. G., Gallet Y., Lopes F., Kovaltsov G. A., Hulot G., 2016, A\&A, 587, A150

Vasiliev S. S., Durgachev V. A., 2002, Ann. Geophys., 20, 115

Wagner G. et al., 2001, Geophys. Res. Lett., 28, 303

Weiss N. O., Tobias S. M., 2016, MNRAS, 456, 2654

This paper has been typeset from a $\mathrm{T}_{\mathrm{E}} \mathrm{X} / \mathrm{L} \mathrm{T} \mathrm{E} \mathrm{X}$ file prepared by the author. 